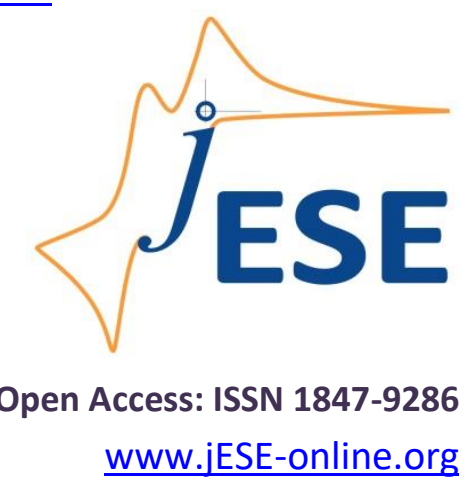

Original scientific paper

\title{
BSA-binding studies of 2- and 4-ferrocenylbenzonitrile: voltammetric, spectroscopic and molecular docking investigations
}

\author{
Hacen Benamara, Touhami Lanez ${ }^{\bowtie}$ and Elhafnaoui Lanez \\ University of El Oued, Chemistry Department, VTRS Laboratory, B.P.789, 39000, El Oued, Algeria \\ Corresponding author: ${ }^{凶}$ lanezt@gmail.com; Tel.: +213-661655550
}

Received: May 29, 2020; Revised: June 11, 2020; Accepted: June 15, 2020

\begin{abstract}
The binding affinity of 2-ferrocenylbenzonitrile (2FBN) and 4-ferrocenylbenzonitrile (4FBN) with bovine serum albumin (BSA) has been investigated by cyclic voltammetry, absorption spectroscopy and molecular modelling techniques. The results indicated that both of the two derivatives could bind to BSA and cause conformational changes with the order $2 F B N>$ $4 F B N$. The voltammetric behavior of $2 F B N$ and $4 F B N$ before and after the addition of BSA suggests that the electrochemical reaction is kinetically controlled by the diffusion step and demonstrated that diffusion coefficients of 2FBN-BSA and 4-FBN-BSA complexes are lower than that of free compounds. Molecular docking suggested that the binding mode of the two compounds to BSA is of hydrophobic and hydrogen bond interactions, moreover the ligand $2 F B N$ additionally show a $\pi$-cation interaction.
\end{abstract}

\section{Keywords}

Cyclic voltammetry; ferrocene derivatives; binding constant; interactions; modelling; in silico; in vitro

\section{Introduction}

Nitriles and cyanides are compounds containing a $-\mathrm{CN}$ functional group in their molecular structure. In nitriles the -CN functional group is attached to an organic structure [1], but in cyanides, it is attached to an inorganic compound [2]. Cyanides are toxic because they denote the highly toxic inorganic salts of hydrogen cyanide, while nitriles are not toxic because they do not release cyanide ions $[3,4]$. The non-toxic properties of nitriles encouraged researchers to study their pharmacochemistry as potential drugs. The prevalence of nitrile-containing drugs shows the biocompatibility of the nitrile functionality $[5,6]$. Recently many pharmaceuticals drugs containing nitriles are either prescribed for many different types of diseases or are in clinical trial [6].

Nitrile groups are usually known as hydrogen bond acceptors [7-9], many studies show the formation of hydrogen bonding between the nitrogen atom of the nitrile group and amino acids of 
protein backbone [10-13]. The bonds are often established between the nitrile and the expected hydrogen bond of the donor serine or arginine amino acids.

Serum albumin is the most important protein in blood plasma and plays a vital role in the transport and distribution of metals, fatty acids, hormones, and renders toxins harmless by transporting them to disposal sites. In addition, BSA binds to variety drugs at multiple sites in the body vascular system [14-16].

BSA interactions with small molecules have become increasingly important in pharmacochemistry and are commonly used as key steps in the construction of medicinal compounds [17-21]. The research in this field provides strong support for BSA-binding studies and a deeper understanding of the way medicaments target and bind receptors [22,23]. BSA interactions with small molecules also help in understanding the toxicity, pharmacokinetics, biochemistry, pharmacodynamics, and distribution of molecules in the organism. Thus, the investigation of BSA interactions with small molecules has been an important analysis in medicinal chemistry and clinical medicine [24].

The pharmacochemistry of ferrocene derivatives has attracted the attention of many scientists, and their study has been encouraged by potential biological applications [25-27]. Many ferrocene derivatives that have been studied in the last decade show important biological activities, such as cytotoxic [28-30], antimicrobial [31,32] and antitumor [33-38] activities.

The incorporation of the ferrocenyl moiety into the structure of biologically active molecules may lead to the increase of their biological activities, based on the fact that nitriles-containing ferrocenes are expected to be more pharmacologically active than free nitriles. In this context the present study surveys the interaction of two nitrile-containing ferrocene with BSA by focusing on the roles of the $\mathrm{CN}$ functional groups. The study was carried out in $0.1 \mathrm{M} 90 \% \mathrm{DMF} /$ buffer phosphate solution at $\mathrm{pH} 7.4$ using voltammetric, spectroscopic and molecular docking techniques.

\section{Experimental}

\section{Synthesis}

2-ferrocenylbenzonitrile (2FBN) and 4-ferrocenylbenzonitrile (4FBN) addressed in this work are shown in Figure $1 \mathrm{a}$ and $1 \mathrm{~b}$, and were synthesised by the coupling reaction between ferrocene and the diazonium salts of the corresponding cyanoaniline, as reported previously by our group [39]. The crystal structure of BSA was taken from protein databank (https://www.rcsb.org/, PDB ID: 4f5s) (Figure 1c).

a

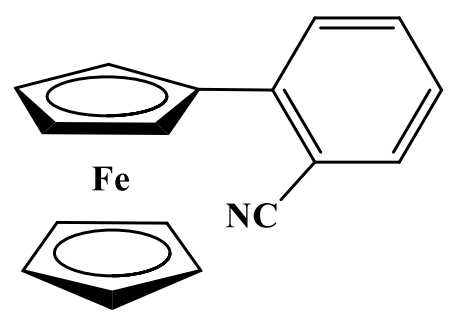

b

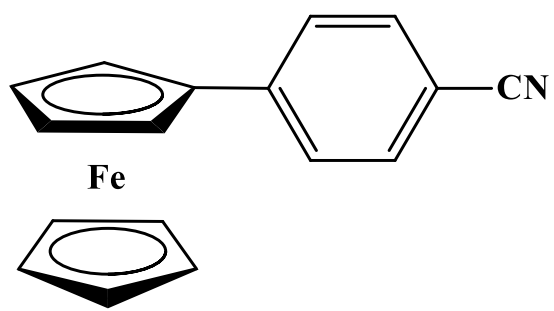

C

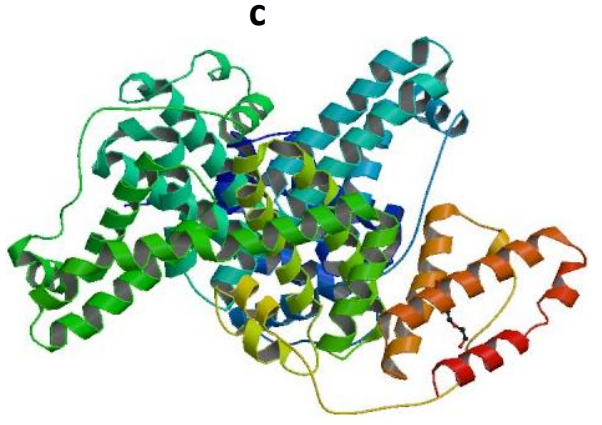

Figure 1. Chemical structures of (a) 2-ferrocenylbenzonitrile, (b) 4-ferrocenylbenzonitrile and (c) structure of BSA (ID: 4f5s)

\section{Chemicals}

All reagents and solvents were of analytical grade and obtained from commercial sources and used without further purification. BSA was obtained from Merck and used as received, while its 
concentration was determined by the extinction coefficient of $44300 \mathrm{M}^{-1} \mathrm{~cm}^{-1}$ at $280 \mathrm{~nm}$ [40]. All stock solutions were used within 5 days after preparation and stored at $4{ }^{\circ} \mathrm{C}$ until use. The phosphate buffer solution was prepared using sodium dihydrogen phosphate and disodium hydrogen phosphate (Sigma Aldrich) and double-distilled water. The physiological pH (pH 7.4) was maintained by this phosphate buffer. N,N-Dimethylformamide (DMF) (HPLC-grade; Sigma-Aldrich) was used as the solvent in voltammetric and spectroscopic assays. Tetrabutylammonium tetra-fluoroborate $\left(\mathrm{Bu}_{4} \mathrm{NBF}_{4}\right)$ (electrochemical grade $99 \%$; Sigma-Aldrich) was used as the supporting electrolyte. Nitrogen gas was provided from a cylinder (research grade (99.99 \%); Linde gaz Algérie).

\section{Materials and measurements}

Voltammetric assays were performed using a PGZ301 voltammeter running on VoltaMaster $4 \mathrm{~V}$ 7.08 software (Radiometer Analytical SAS, France). The concentration of the supporting electrolyte was kept at $0.1 \mathrm{M}$ all time. The air was removed from the solution by bubbling nitrogen gas through it. Experiments were run in a three-electrode electrochemical cell containing a glassy carbon (GC) working electrode with a geometric area of $0.013 \mathrm{~cm}^{2}$, a platinum wire as counter (auxiliary) electrode and $\mathrm{Hg} / \mathrm{Hg}_{2} \mathrm{Cl}_{2}$ paste covered wire as reference electrode.

Absorption spectra measurements were conducted on a UV-Vis spectrometer, (Shimadzu 1800, Japan), using the cell of length $1 \mathrm{~cm}$.

Chemical structures of 2FBN and 4FBN were optimized by Gaussian 09 program package [41], using density functional theory (DFT) and the B3LYP level of theory $[42,43]$ with $6-311++G(d, p)$ basis set.

The molecular docking studies were done using AutoDock 4.2 docking software [44,45], all docking studies were executed on a Pentium $2.20 \mathrm{GHz}$ and RAM 4.00 Go microcomputer MB memory with windows 10 operating system.

\section{Results and discussion}

\section{Cyclic voltammetric study}

BSA-2FBN and BSA-4FBN complexes in $0.1 \mathrm{M} 90 \% \mathrm{DMF} /$ buffer phosphate solution at $\mathrm{pH} 7.4$ were used. Various concentrations of BSA were added into $12 \mathrm{ml}$ solution of $100 \mu \mathrm{M}$ of the ligand solutions, and the voltammograms were recorded in the potential range of 0.1 to $0.8 \mathrm{~V} \mathrm{vs} . \mathrm{Hg} / \mathrm{Hg}_{2} \mathrm{Cl}_{2}$ at $28 \pm 1{ }^{\circ} \mathrm{C}$.

Many studies on the interaction of BSA with small molecules in this potential range have been carried out using cyclic voltammetry techniques, and all these studies have shown that BSA does not show any adsorption on the bare electrode surface at this potential range [46-50]. Adsorption of BSA can only appear at negative potential [51].

The cyclic voltammograms (Figure 2) of 2FBN and 4FBN at different concentrations of BSA show respectively oxidation and reduction maximums in a reversible electrochemical process. Addition of an increasing amount of BSA solution results in a decrease in peak current height with a positive shift in peak potential position. This decrease in anodic peak current height is exploited for the calculation of the binding parameters.

The binding constant, $K_{b}$, was calculated from the observed cyclic voltammetry data, using the following equation [52]:

$$
\log \frac{1}{c_{\mathrm{BSA}}}=\log K_{\mathrm{b}}+\log \frac{i}{i_{0}-i}
$$


where $C_{B S A}$ is BSA concentration, $K_{b}$ represents the binding constant, while $i_{0}$ and $i$ indicate the anodic peak current density of the free and BSA-bound compounds, respectively.
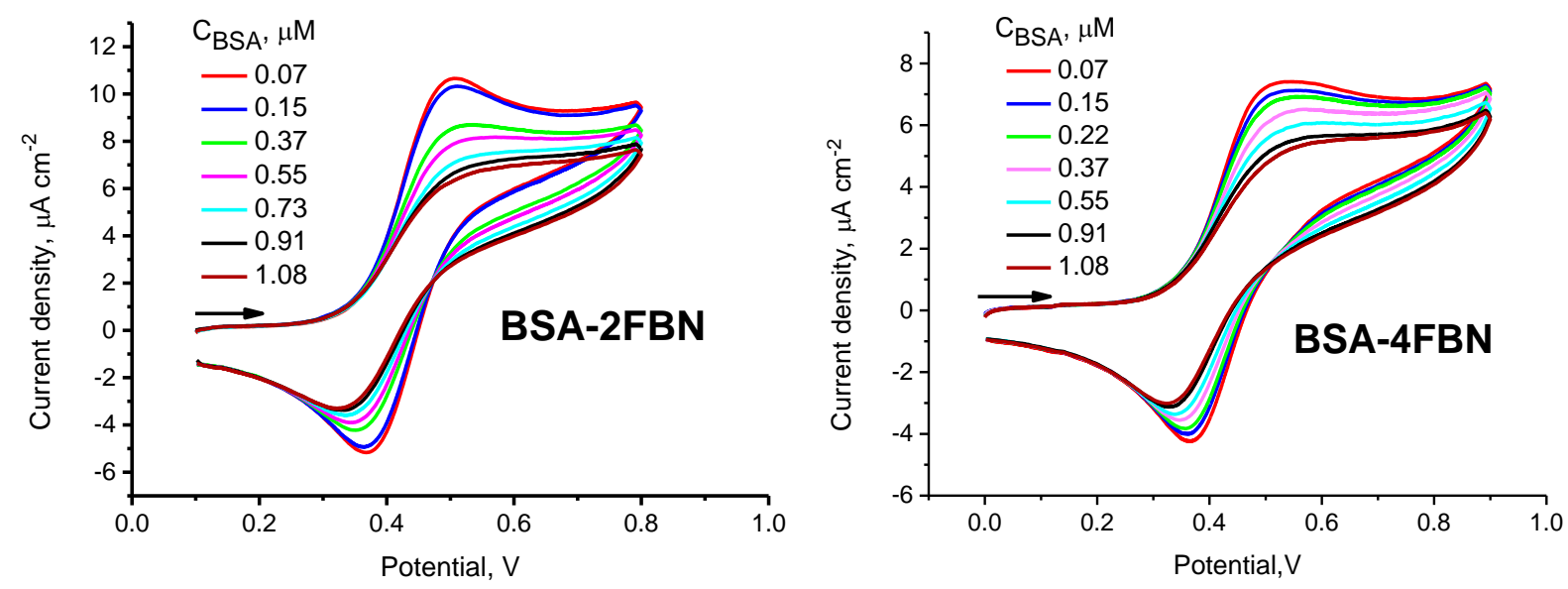

Figure 2. Cyclic voltammograms of $B S A-2 F B N$ and $B S A-4 F B N$ complexes at different concentration of BSA. $2 F B N$ and $4 F B N$ concentrations were kept at $100 \mu M$

Obviously, $K_{\mathrm{b}}$ can be determined from the intercept of the plot of $\log \left(1 / C_{\mathrm{BSA}}\right)$ vs. $\log \left(i /\left(i_{0}-i\right)\right)$. These plots are for $2 \mathrm{FBN}$ and $4 \mathrm{FBN}$ represented in Figure 3, from which the values of binding constants were determined as $7.05 \times 10^{5} \mathrm{M}^{-1}$ for $2 \mathrm{FBN}$ and $3.44 \times 10^{5} \mathrm{M}^{-1}$ for $4 \mathrm{FBN}$. The binding free energy changes calculated using the equation $\Delta G=-n R T \ln K_{\mathrm{b}}$ are found equal to -33.94 and -32.14 $\mathrm{kJ} \mathrm{mol}^{-1}$, respectively. The order of magnitude and the sign of the obtained binding free energy indicate respectively the electrostatic mode and the spontaneity of interactions between the compounds and BSA.
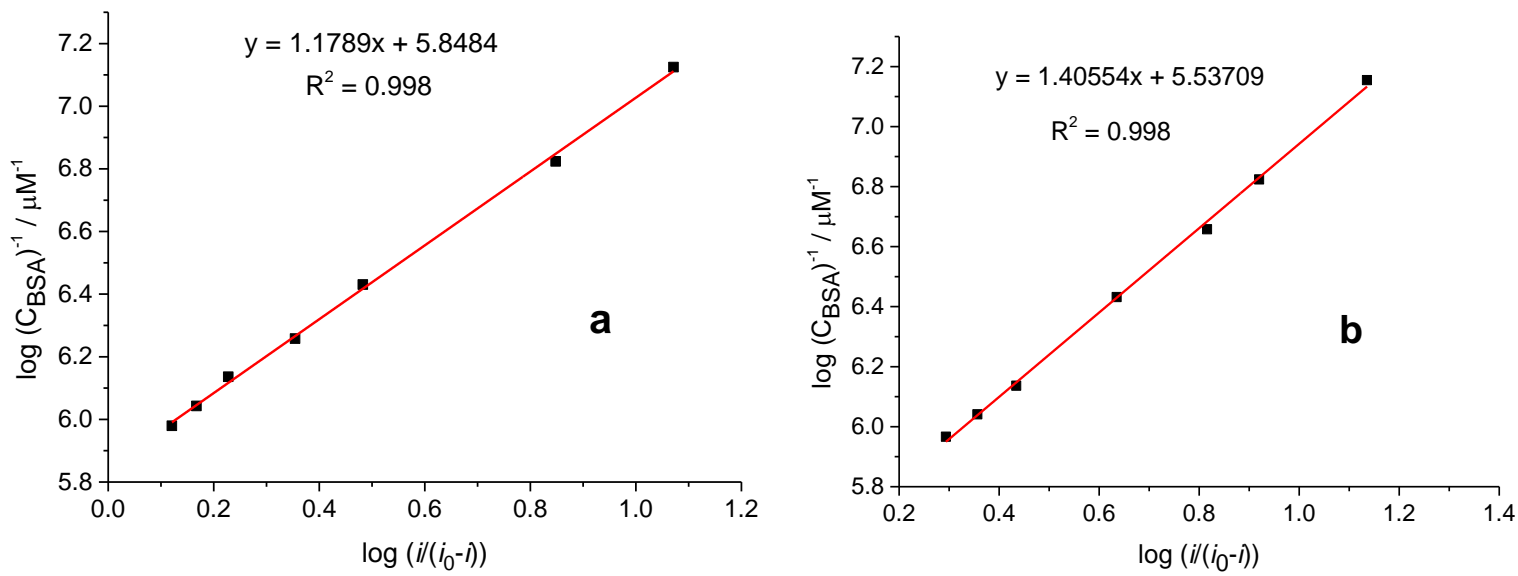

Figure 3. Plots of $\log \left(C_{B S A}\right)^{-1}$ vs. $\log \left(i /\left(i_{0}-i\right)\right)$ used to calculate BSA binding constants: (a) $2 F B N$, (b) $4 F B N$

\section{Ratio of binding constants}

The ratio of the binding constants of the reaction of the reduced form FBN (FBN represents $2 F B N$ or $4 \mathrm{FBN}$ ) with $\mathrm{BSA}$ to that of the oxidized form $[\mathrm{FBN}]^{+}$, could be calculated from the voltammograms of Figure 4, which represent the cyclic voltammograms of $100 \mu \mathrm{M}$ solution of $2 \mathrm{FBN}$ and $4 \mathrm{FBN}$ in the absence and presence of $0.37 \mu \mathrm{M}$ of BSA. The shift in the anodic and cathodic peak potential values caused by the addition of BSA can be used to calculate the ratio of binding constants [53]. 

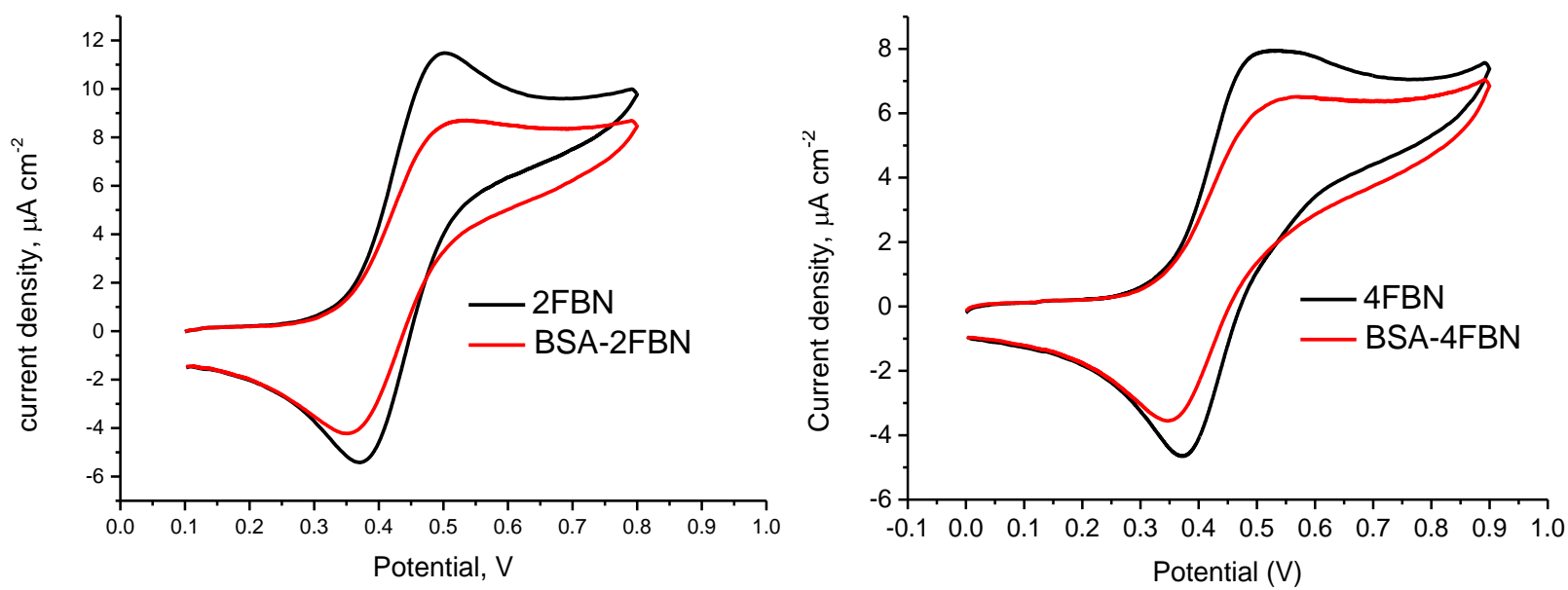

Figure 4. Cyclic voltammograms of the free compounds $(100 \mu \mathrm{M})$ and their BSA complexes $(0.37 \mu \mathrm{M})$, scan rate $100 \mathrm{mV} \mathrm{s}^{-1}$

In such case when both the anodic and cathodic peak potential values are shifted upon the addition of BSA, the following equilibriums can be applied [54]:

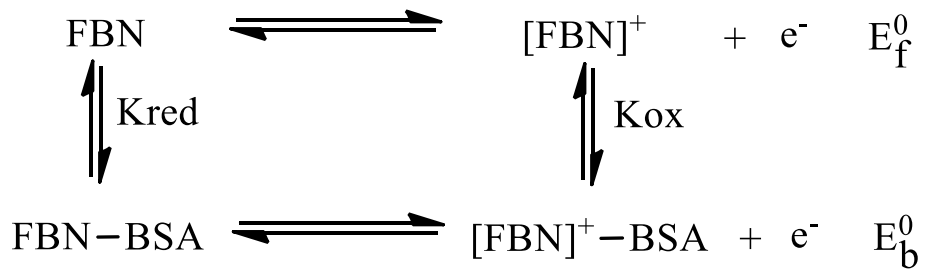

Figure 5. Redox process of studied compounds with BSA, FBN represents $2 F B N$ or $4 F B N$.

The application of the Nernst relation to the equilibriums of Figure 5 produces the following equation:

$$
\Delta E^{0}=E_{\mathrm{b}}^{0}-E_{\mathrm{f}}^{0}=E^{0}(\mathrm{FBN}-\mathrm{BSA})-E^{0}(\mathrm{FBN})=0.06 \log \frac{K_{\mathrm{red}}}{K_{\mathrm{ox}}}
$$

In equation (2), $E_{\mathrm{f}}{ }^{0}$ and $E_{\mathrm{b}}{ }^{0}$ are the formal potentials of the $\mathrm{FBN}^{+} / \mathrm{FBN}$ couple of free and BSAbound compounds, respectively. The formal potential shift $\Delta E^{0}$ calculated on the basis of the voltammograms of Figure 4, are summarized in Table 1. The ratios of the binding constants were calculated from equation (2) by replacing $\Delta E^{0}$ taken from Table 1.

Table 1. Electrochemical data of free and BSA-bound $2 F B N$ and $\triangle F B N$ used to calculate the ratio of the binding constants.

\begin{tabular}{|c|c|c|c|c|c|}
\hline Sample code & $E_{\mathrm{pa}} / \mathrm{V}$ & $E_{\mathrm{pc}} / \mathrm{V}$ & $E^{0} / \mathrm{V}$ & $\Delta E^{0} / \mathrm{mV}$ & $K_{\text {red }} / K_{\text {ox }}$ \\
\hline $2 \mathrm{FBN}$ & 0.502 & 0.369 & 0.4355 & \multirow{2}{*}{3.5} & \multirow{2}{*}{1.14} \\
\hline $2 \mathrm{FBN}-\mathrm{BSA}$ & 0.527 & 0.351 & 0.439 & & \\
\hline $4 \mathrm{FBN}$ & 0.538 & 0.374 & 0.456 & \multirow{2}{*}{4.0} & \multirow{2}{*}{1.16} \\
\hline 4FBN-BSA & 0.572 & 0.348 & 0.46 & & \\
\hline
\end{tabular}

The obtained ratios of the binding constants indicate that the reduced form of both $2 \mathrm{FBN}$ and 4FBN bind slightly stronger to BSA than their oxidized forms.

\section{Diffusion coefficients}

The diffusion coefficients of the free and BSA-bound 2FBN and 4FBN compounds were obtained from their electrochemical behavior represented in Figures 6 and 7. These cyclic voltammograms were obtained by varying the potential scan rates of $100 \mu \mathrm{M}$ of the free compounds in the absence 
and presence of $0.91 \mu \mathrm{M}$ of BSA. All the voltammograms present well-defined stable redox peaks attributed to the redox process of $2 \mathrm{FBN}$ and $4 \mathrm{FBN}$.
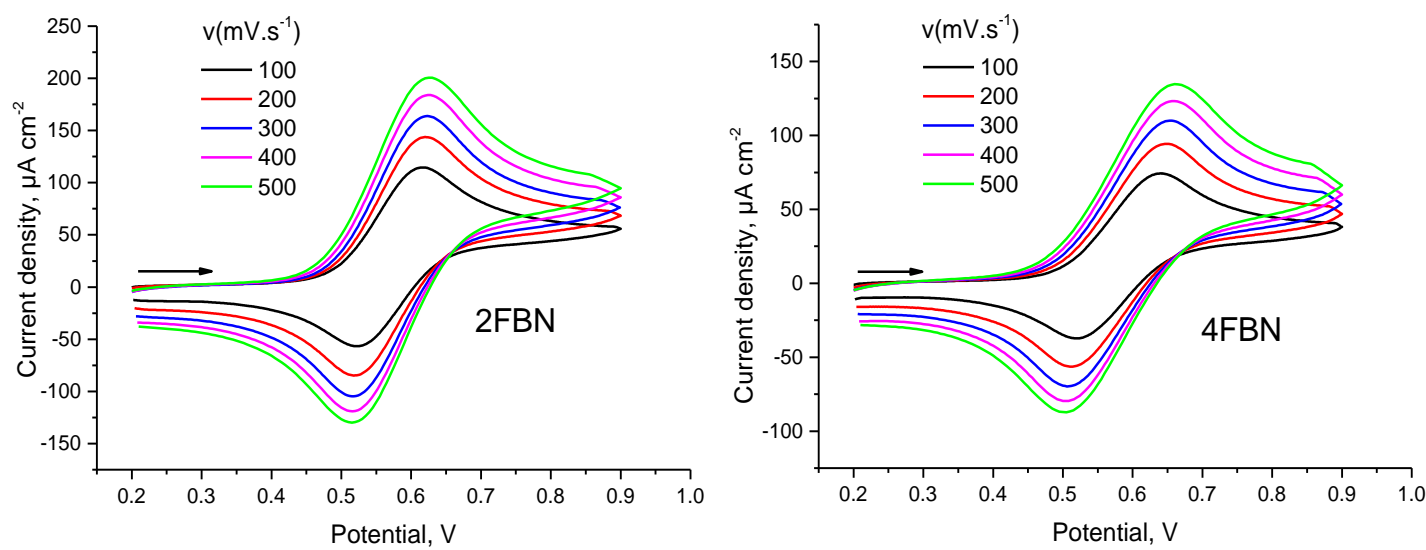

Figure 6. Cyclic voltammetric behavior of $100 \mu \mathrm{M}$ of $2 F B N$ and $4 F B N$ in $0.1 \mathrm{M} 90 \% \mathrm{DMF} /$ buffer phosphate solution at different scan rates.
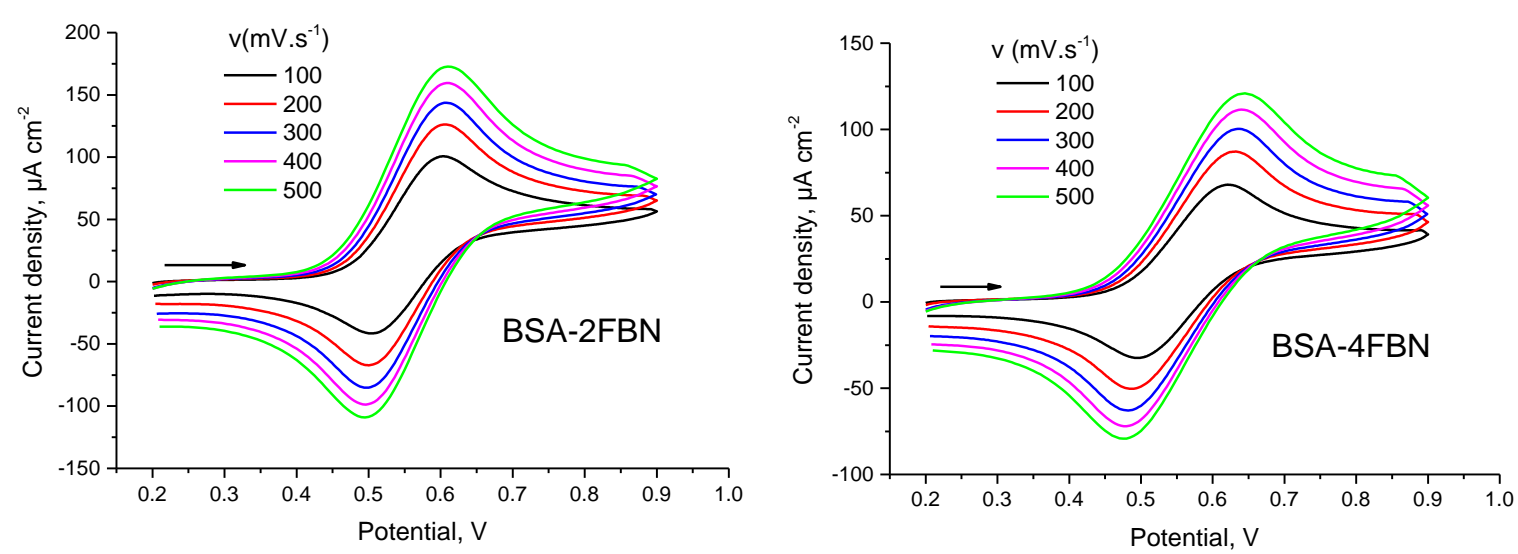

Figure 7. Cyclic voltammetric behavior of $0.91 \mu \mathrm{M}$ of BSA-2FBN and BSA-4FBN in $0.1 \mathrm{M} 90 \%$ $D M F /$ buffer phosphate solution at different scan rates

Diffusion coefficients of FBN-BSA in the solution with an excess of BSA were calculated from the voltammograms of Figures 6 and 7 using the following Randles-Ševčik equation [55]:

$$
i_{\mathrm{pa}}=2.69 \times 10^{5} n^{\frac{3}{2}} S C D^{\frac{1}{2}} v^{\frac{1}{2}}
$$

In equation (3), $i_{\text {pa }}$ represents the anodic peak current (A), $n$ is the number of electrons participated in the oxidation process, $S$ is the surface of the working electrode $\left(\mathrm{cm}^{2}\right), C$ is the concentration of the electroactive compounds (mol cm$\left.{ }^{-3}\right), D$ is the diffusion coefficient $\left(\mathrm{cm}^{2} \mathrm{~s}^{-1}\right)$, and $v$ is the scan rate $\left(\mathrm{V} \mathrm{s}^{-1}\right)$. The plots of $i_{\mathrm{pa}} v s . v^{1 / 2}$ in Figure 8 suggest that the oxidation reaction is diffusion controlled. The diffusion coefficients of the free and BSA-bound compounds were calculated from the slopes of linear regressions of the plots of $i_{\mathrm{pa}} v s . v^{1 / 2}$. The lower diffusion coefficients of the bound compounds as compared to the free once, further confirm the interaction between the studied compounds and BSA (Table 2).

Table 2. Diffusion constant values of the free and BSA bound form of $2 F B N$ and $4 F B N$.

\begin{tabular}{cccc}
\hline Sample code & Equation & $R^{2}$ & $D \times 10^{6} / \mathrm{cm}^{2} \mathrm{~s}^{-1}$ \\
2FBN & $\mathrm{y}=6.94 \mathrm{x}+44.25$ & 0.999 & 3.94 \\
2FBN-BSA & $\mathrm{y}=5.78 \mathrm{x}+42.97$ & 0.999 & 2.73 \\
4FBN & $\mathrm{y}=4.89 \mathrm{x}+24.63$ & 0.999 & 1.96 \\
4FBN-BSA & $\mathrm{y}=4.22 \mathrm{x}+26.40$ & 0.999 & 1.46 \\
\hline
\end{tabular}



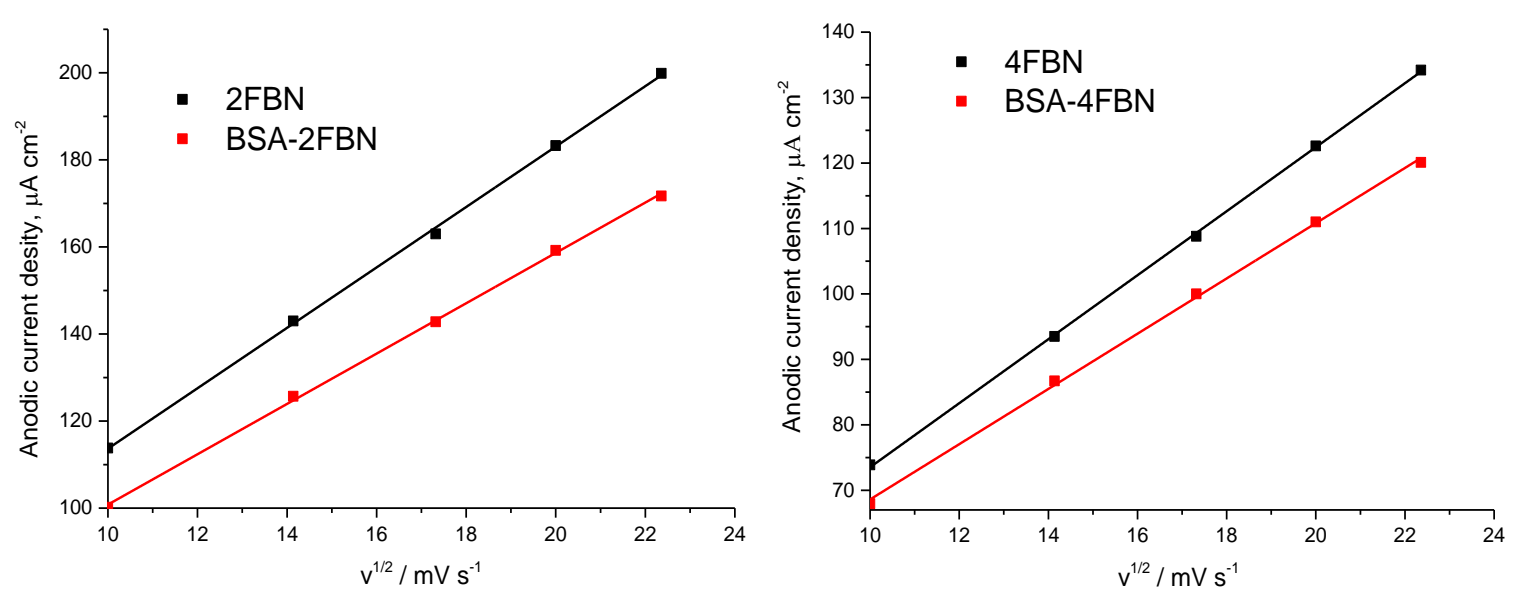

Figure 8. $i_{p a}$ Vs. $v^{1 / 2}$ plots of $100 \mu \mathrm{M} 2 F B N$ and $4 F B N$ in the absence and in presence of $0.91 \mu \mathrm{M}$ $B S A$ based on voltammograms in Figures 6 and 7.

\section{Absorption spectroscopic studies}

The interactions of 2FBN and 4FBN with BSA were also studied by absorption spectroscopic titration. The purpose of this study was to validate the results obtained from cyclic voltammetry assays. The experiments were carried out with $0.1 \mathrm{M} 90 \% \mathrm{DMF} /$ buffer phosphate solution of $\mathrm{pH} 7.4$. Incremental portions of BSA solution from 0.34 to $0.70 \mu \mathrm{M}$ for $2 \mathrm{FBN}$ and from 0.15 to $2.67 \mu \mathrm{M}$ for $4 F B N$ were added to the same solution containing $0.5 \mathrm{mM}$ of $2 \mathrm{FBN}$ and $2 \mathrm{mM}$ of $4 \mathrm{FBN}$. The obtained mixture was scanned in the range of 300-600 $\mathrm{nm}$. BSA does not show any absorption at this wavelength, while the strong peak which appeared at $434.5 \mathrm{~nm}$ (due to $\pi \rightarrow \pi^{*}$ transition in the conjugated ring of ferrocene moiety) lowered in intensity upon continuous addition of BSA to 2FBN and 4FBN (Figure 9).
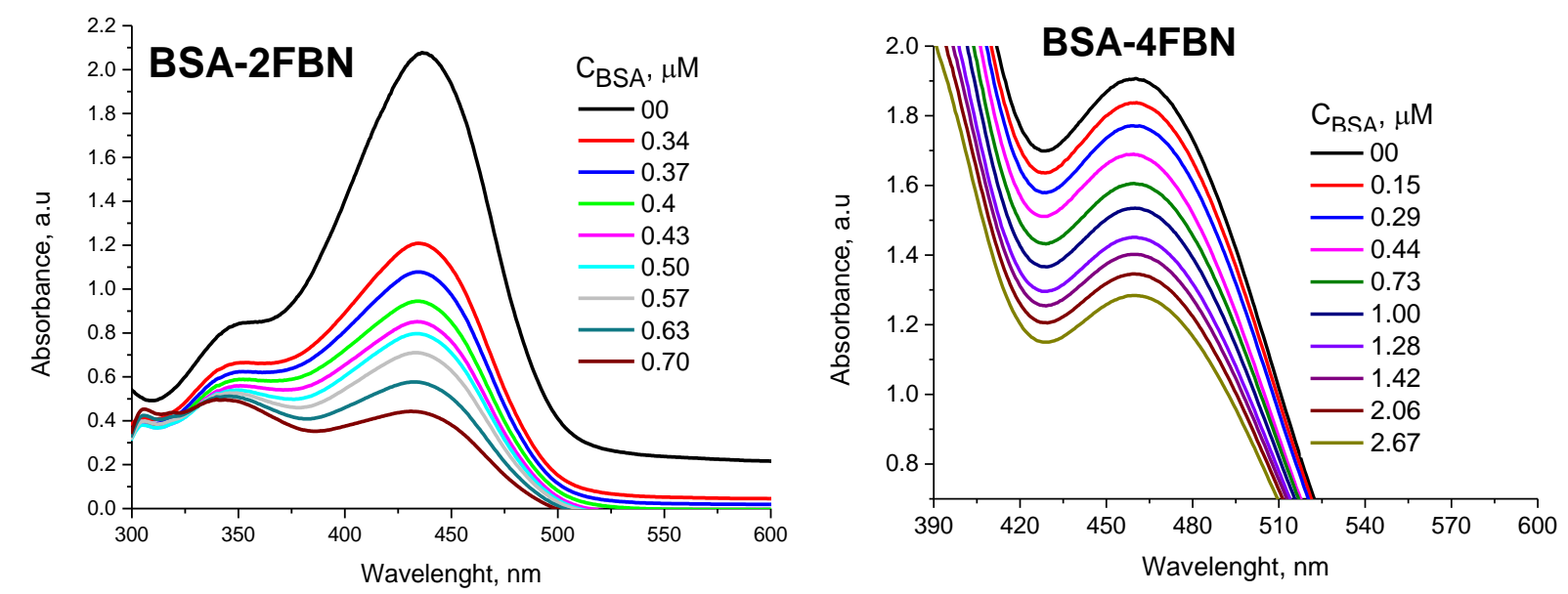

Figure 9. Absorbance spectra of $2 F B N-B S A$ and $4 F B N-B S A$ complexes. $2 F B N$ and $4 F B N$ concentrations were kept respectively at 0.5 and $2 \mathrm{mM}$

The binding constant $K_{\mathrm{b}}$ was evaluated from the absorption data according to Benesi-Hildebrand equation [56]:

$$
\frac{A_{0}}{A-A_{0}}=\frac{\varepsilon_{\mathrm{f}}}{\varepsilon_{\mathrm{b}}-\varepsilon_{\mathrm{f}}}+\frac{\varepsilon_{\mathrm{f}}}{\varepsilon_{\mathrm{b}}-\varepsilon_{\mathrm{f}}} \frac{1}{K_{\mathrm{b}} C_{\mathrm{BSA}}}
$$

In equation (4), $A_{0}$ and $A$ are absorbencies of the ligands and their complexes with BSA, respectively, while $\varepsilon_{\mathrm{f}}$ and $\varepsilon_{\mathrm{b}}$ are their extinction coefficients. The plot of $A_{0} /\left(A_{0}-A\right) v s .1 / C_{B S A}$ gave a 
slope of $\varepsilon_{\mathrm{f}} /\left(\varepsilon_{\mathrm{b}}-\varepsilon_{\mathrm{f}}\right) K_{\mathrm{b}}$, and intercept equal to $\varepsilon_{\mathrm{f}} /\left(\varepsilon_{\mathrm{b}}-\varepsilon_{\mathrm{f}}\right)$, where $K_{\mathrm{b}}$ is the ratio of the slope to the intercept (Figure 10). The value of $K_{\mathrm{b}}$ has been determined to be $7.18 \times 10^{5}$ for 2 FBN-BSA and $2.79 \times 10^{5} \mathrm{M}^{-1}$ for $4 \mathrm{FBN}-\mathrm{BSA}$. The corresponding free binding energies calculated using the equation $\Delta G=-n R T \ln K_{\mathrm{b}}$ were equal to -33.77 and $-31.40 \mathrm{~kJ} \mathrm{~mol}^{-1}$, respectively. These values are in good agreement with those obtained from cyclic voltammetry experiments.
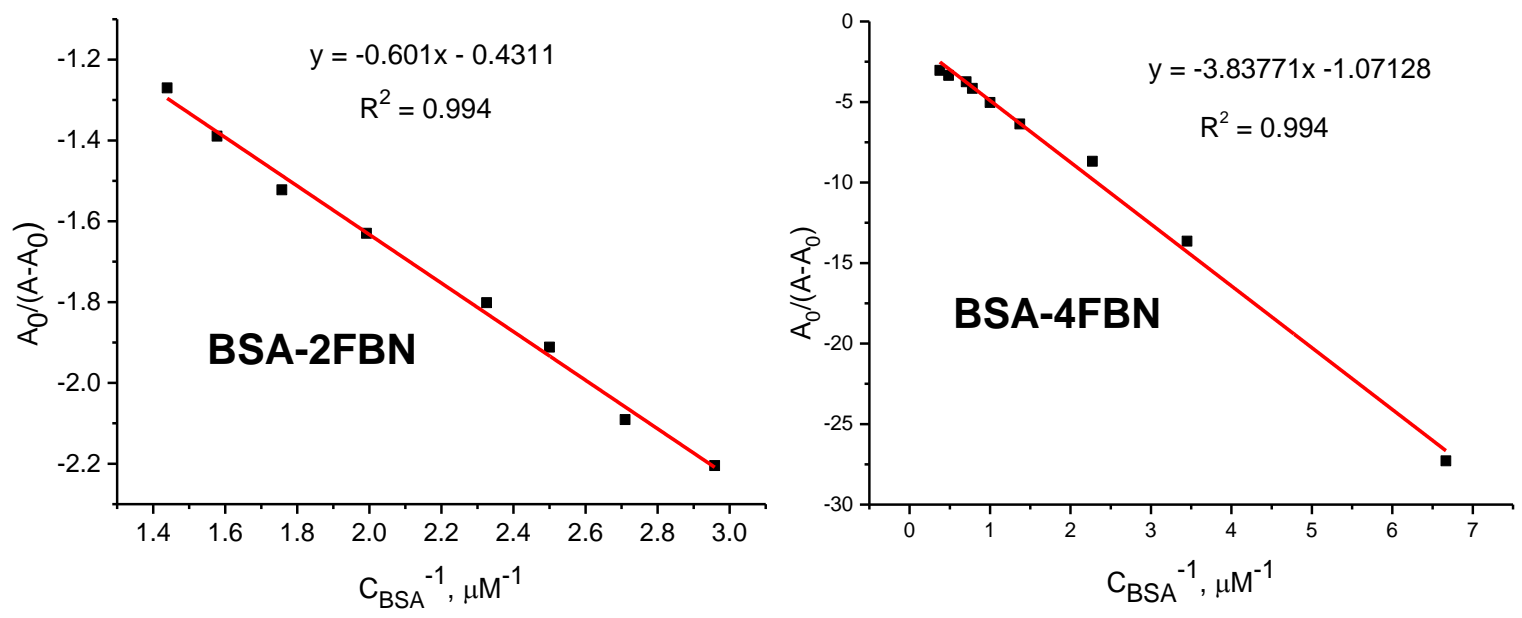

Figure 10. Plots of $A_{0} /\left(A_{0}-A\right)$ vs. $C_{B S A}{ }^{-1}$ used to calculate the binding constants of $B S A-2 F B N$ and $B S A-4 F B N$

\section{Docking setup}

\section{Geometry optimization}

Density functional theory (DFT) was used for the optimisation without imposing any symmetry constraints and calculations were realized with the Gaussian 09 package. The exchange functional of Becke, and the correlation functional of Lee, Yang and Parr (B3LYP) were employed with 6$311++G(d, p)$ basis set. The optimized structures of the compounds are depicted in Figure 11.

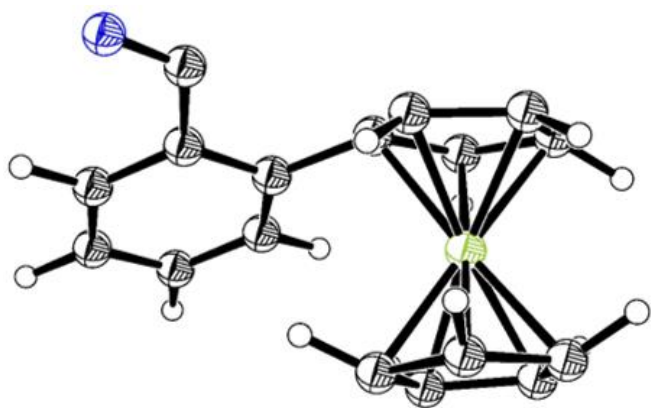

$2 \mathrm{FBN}$

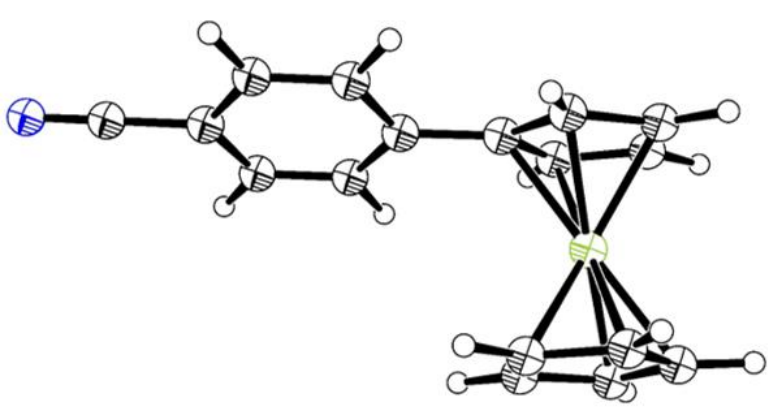

$4 \mathrm{FBN}$

Figure 11. The optimized structures of $2 F B N$ and $4 F B N(O R T E P$ View $03, V 1.08) ;$ color codes are carbon (grey), hydrogen (white), nitrogen (blue), iron (green).

Molecular docking studies

In order to confirm and interpret the results of cyclic voltammetric and spectrophotometric measurements and recognize the way in which $2 \mathrm{FBN}$ and $4 \mathrm{FBN}$ bind to BSA, semi flexible docking was carried out using AutoDock 4.2 along with the AutoDock Vina software. The crystal structure of BSA was taken from the protein databank (https://www.rcsb.org/, PDB ID: 4f5s). The PDB file was imported into AutoDock Tools, all hydrogen atoms and gassier charges were added. During all docking process, BSA kept rigid while all the bonds of the ligands were set free. The grid map with $0.375 \AA$ spacing and $126 \times 70 \times 100$ points were generated. The docking experiment comprised of 100 docking 
runs with 150 individuals and 2.500 .000 energy evaluations. Lamarckian genetic algorithm was used in the docking, and other parameters were set as default. The stable conformation which corresponds to the lowest binding energy was used for docking analysis. The visualization of the interaction was generated with PLIP web service (protein Ligand Interaction Profiler).

Results from molecular docking suggest that hydrogen bonding, hydrophobic forces and $\pi$-cation interaction are involved in the binding process. Figure 12 illustrates the interaction of $2 \mathrm{FBN}$ and $4 \mathrm{FBN}$ with the nearby residues in the active site of BSA.

The interacting residues, distances, and type of interactions are summarised in Tables 3 and 4.

Table 3. Hydrophobic interactions between the ligands $2 F B N$ and $4 F B N$ with BSA.

\begin{tabular}{cccc}
\hline Interaction type & Residue & Amino acid & Distance, $\AA$ \\
\hline \multirow{3}{*}{ BSA-BSA } & $80 \mathrm{~A}$ & LEU & 2.85 \\
\cline { 2 - 4 } & $81 \mathrm{~A}$ & ARG & 3.45 \\
\cline { 2 - 4 } & $88 \mathrm{~A}$ & ALA & 3.72 \\
\hline & $115 \mathrm{~B}$ & LEU & 3.99 \\
\hline & $115 \mathrm{~B}$ & LEU & 3.57 \\
\cline { 2 - 4 } BSA-4FBN & 122B & LEU & 3.14 \\
\cline { 2 - 4 } & 136B & LYS & 3.94 \\
\cline { 2 - 4 } & 137B & TYR & 3.15 \\
\cline { 2 - 4 } & 140B & GLU & 3.68 \\
\cline { 2 - 4 } & 141B & ILE & 3.58 \\
\hline
\end{tabular}

Table 4. Hydrogen bonds between the ligands $2 F B N$ and $4 F B N$ and BSA.

\begin{tabular}{ccccc}
\hline Interaction type & Residue & Amino acid & Distance H-A, $\AA$ & Distance D-A, $\AA$ \\
\hline \multirow{2}{*}{ 2FBN-BSA } & $81 \mathrm{~A}$ & ARG & 3.00 & 3.45 \\
\cline { 2 - 5 } & $82 \mathrm{~A}$ & GLU & 2.04 & 3.05 \\
\cline { 2 - 5 } & $82 \mathrm{~A}$ & $\mathrm{GLU}$ & 3.22 & 3.71 \\
\hline 4FBN-BSA & $137 \mathrm{~B}$ & TYR & 2.38 & 3.32 \\
\hline
\end{tabular}

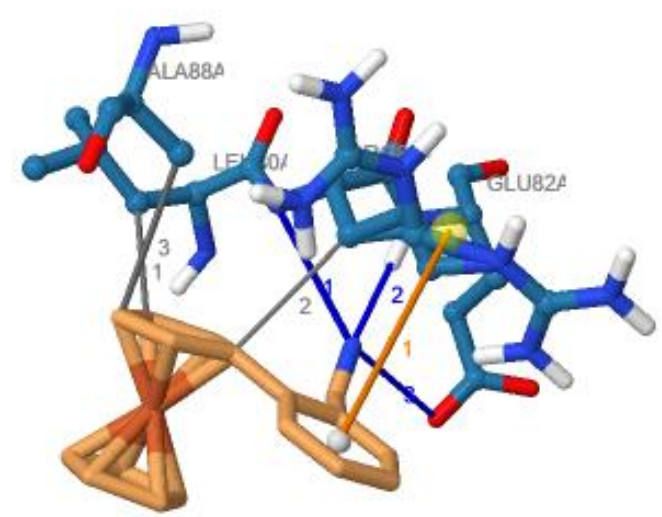

BSA-2FBN

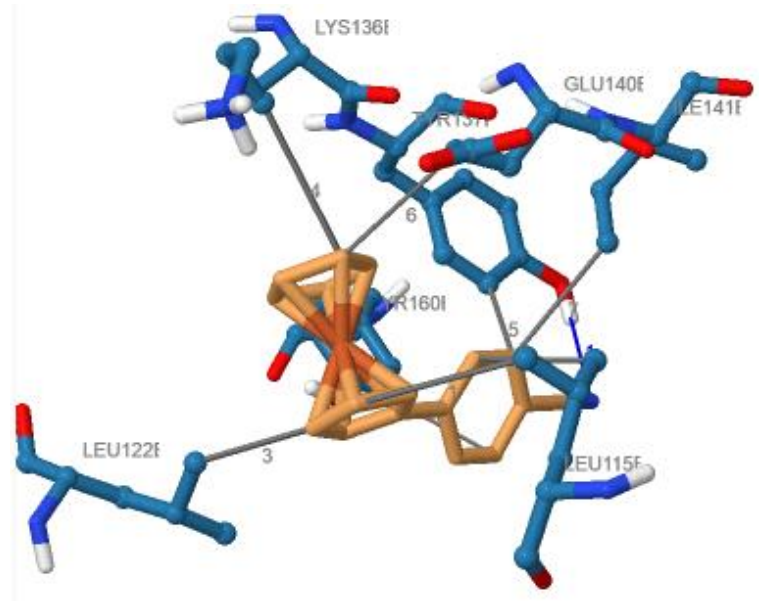

BSA-4FBN

Figure 12. Best docking poses for BSA-2FBN and BSA-4FBN generated with PLIP web service illustrating the hydrophobic and H-bons interactions. Elements colors: hydrogen, oxygen, nitrogen, and iron are represented in white, red, blue and brown, respectively Color code: Hydrogen bonds: bleu lines, hydrophobic interactions: gray lines, $\pi$-Cation interactions: beige lines. 
The compound 2FBN formed three hydrogen bonds between amino acid residues Arg- 81 and Glu-82 as donors and the polar groups of the ligand - nitrile group. 4FBN formed only one hydrogen bond between the residue Tyr-137 as acceptor and the nitrogen of the 4FBN as a donor, table 4. The distances in table 4 are between hydrogen and the receptor atoms $(\mathrm{H}-\mathrm{A})$, and between donor and receptor atoms (D-A). Furthermore, for the complex 2FBN-BSA, molecular docking results also suggested a $\pi$-cation interaction between the positively charged amino acid residue Arg-81 and the benzene ring within a distance of $5.01 \AA$.

The ligand 4FBN interacted through hydrophobic interactions with the residues Leu-115, Leu122, Lys-136, Tyr-137, Glu-140, Ile-141, and Tyr-160,

The binding free energy of the docked structure of 2FBN and 4FBN ligands with BSA was found to be -32.89 and $-31.26 \mathrm{~kJ} \mathrm{~mol}^{-1}$, respectively. The binding constant $K_{\mathrm{b}}$ calculated using the equation $\Delta G=-n R T \ln K_{\mathrm{b}}$ was found to be $5.77 \times 10^{5}$ and $2.99 \times 10^{5} \mathrm{~mol}^{-1}$, respectively. These results are supported by the absorption spectroscopy and cyclic voltammetry experiments. Overall, molecular docking results are in good agreement with the data obtained from experimental assays.

\section{Conclusions}

In the present work, we applied experimental and theoretical methods for the determination of binding proprieties of two nitrile-containing ferrocenes with BSA. Binding free energies for the interaction of $2 \mathrm{FBN}$ and $4 \mathrm{FBN}$ with $\mathrm{BSA}$, obtained from voltammetric experiments, were respectively -33.95 and $-32.14 \mathrm{~kJ} \mathrm{~mol}^{-1}$, and these values are in good agreement with those obtained from adsorption spectroscopic assays $\left(-33.77\right.$ and $\left.-31.40 \mathrm{~kJ} \mathrm{~mol}^{-1}\right)$. The low diffusion coefficient values of the BSA-bound $2 \mathrm{FBN}$ and $4 \mathrm{FBN}$ as compared to the corresponding free $2 \mathrm{FBN}$ and $4 \mathrm{FBN}$, further confirm the formation of the complexes 2FBN-BSA and 4FBN-BSA which diffuses more slowly compared to the free compounds due to their higher molecular weight. Molecular docking study further confirms the obtained experimental results and allows the visualisation of interactions and determination of bonds length formed between the ligands and the amino acid residues of BSA.

Acknowledgements: The authors are grateful to the Algerian Ministry of Higher Education and Research for financial support (project code: B00L01UN390120150001).

\section{References}

[1] Compendium of Polymer Terminology and Nomenclature, IUPAC Recommendations 2008, R. G. Jones, J. Kahovec, R. Stepto, E. S. Wilks, M. Hess, T. Kitayama, W. Val Matanomski (Eds.). With advice from A. Jenkins and P. Kratochvil, The Royal Society of Chemistry, Cambridge, UK, 2009, p. 98.

[2] Nitriles - MeSH - NCBI, (n.d.). https://www.ncbi.nlm.nih.gov/mesh/68009570 (accessed April 22, 2020).

[3] J. Wit, H. Van Genderen, Biochemical Journal 101(3) (1966) 698-706.

[4] D. H. Hutson, E. C. Hoadley, M. H. Griffiths, C. Donninger, Journal of Agricultural and Food Chemistry 18(3) (1970) 507-512.

[5] S. T. Murphy, H. L. Case, E. Ellsworth, S. Hagen, M. Huband, T. Joannides, C. Limberakis, K. R. Marotti, A. M. Ottolini, M. Rauckhorst, J. Starr, M. Stier, C. Taylor, T. Zhu, A. Blaser, W. A. Denny, G. L. Lu, J. B. Smaill, F. Rivault, Bioorganic and Medicinal Chemistry Letters 17(8) (2007) 2150-2155.

[6] F. F. Fleming, L. Yao, P. C. Ravikumar, L. Funk, B. C. Shook, Journal of Medicinal Chemistry 53(22) (2010) 7902-7917.

[7] J-Y. Le Questel, M. Berthelot, C. Laurence, Journal of Physical Organic Chemistry 13(6) (2000) 347358.

[8] C. Laurence, K. A. Brameld, J. Graton, J. Y. Le Questel, E. Renault, Journal of Medicinal Chemistry 52(14) (2009) 4073-4086.

[9] A. Allerhand, P. von Rague Schleyer, Journal of the American Chemical Society 85(7) (1963) 866-870. 
[10] Y. Wang, Y. Du, N. Huang, Future Medicinal Chemistry 10(23) (2018) 2713-2728.

[11] L. Xue, F. Zou, Y. Zhao, X. Huang, Y. Qu, Spectrochimica Acta - Part A: Molecular and Biomolecular Spectroscopy 97 (2012) 858-863.

[12] R. E. Royer, M. Kibirige, C. R. Tafoya, D. L. Vander Jagt, L. M. Deck, Journal of Pharmaceutical Sciences 77(3) (1988) 237-240.

[13] J. T. First, J. D. Slocum, L. J. Webb, Journal of Physical Chemistry B 122(26) (2018) 6733-6743.

[14] T. Peters Jr., All about albumin: biochemistry, genetics, and medical applications, Academic Press Inc., San Diego, California, 1996, p. 382.

[15] M. Fasano, S. Curry, E. Terreno, M. Galliano, G. Fanali, P. Narciso, S. Notari, P. Ascenzi, IUBMB Life 57(12) (2005) 787-796.

[16] P. Lee, X. Wu, Current Pharmaceutical Design 21(14) (2015) 1862-1865.

[17] T. Topală, A. Bodoki, L. Oprean, R. Oprean, Clujul Medical 87(4) (2014) 215-219.

[18] L. Zhang, Q. Y. Cai, Z. X. Cai, Y. Fang, C. S. Zheng, L. L. Wang, S. Lin, D. X. Chen, J. Peng, Molecules 21(12) (2016) 1706. https://doi.org/10.3390/molecules21121706.

[19] Q. Zhang, Y. Ni, RSC Advances 7(63) (2017) 39833-39841.

[20] D. Sood, N. Kumar, G. Rathee, A. Singh, V. Tomar, R. Chandra, Scientific Reports 8 (2018) 16964. doi.org/10.1038/s41598-018-35384-6.

[21] J. Yu, J.-Y. Liu, W.-M. Xiong, X.-Y. Zhang, Y. Zheng, BMC Chemistry 13 (2019). 95. https://doi.org/10.1186/s13065-019-0615-6.

[22] D. Sleep, Expert Opinion on Drug Delivery 12 (2015) 793-812.

[23] G. L. Francis, Cytotechnology 62(1) (2010) 1-16.

[24] A. M. Bagoji, J. I. Gowda, N. M. Gokavi, S.T. Nandibewoor, Journal of Biomolecular Structure and Dynamics 35(11) (2017) 2395-2406.

[25] F. A. Larik, A. Saeed, T. A. Fattah, U. Muqadar, P. A. Channar, Applied Organometallic Chemistry 31(8) (2016), doi.org/10.1002/aoc.3664.

[26] M. Patra, G. Gasser, Nature Reviews Chemistry 1(9) (2017) 0066. https://doi.org/10.1038/s41570017-0066.

[27] M. Patra, G. Gasser, M. Wenzel, K. Merz, J. E. Bandow, N. Metzler-Nolte, Organometallics 29(19) (2010) 4312-4319.

[28] M. Görmen, P. Pigeon, S. Top, E. A. Hillard, M. Huché, C. G. Hartinger, F. de Montigny, M. A. Plamont, A. Vessières, G. Jaouen, ChemMedChem 5 (2010) 2039-2050.

[29] W. I. Pérez, Y. Soto, C. Ortíz, J. Matta, E. Meléndez, Bioorganic and Medicinal Chemistry 23(3) (2015) 471-479.

[30] J. Yong, X. Wang, X. Wu, C. Lu, Journal of Infectious Diseases \& Therapy 5(1) (2017) 1000311. https://doi.org/10.4172/2332-0877.1000311.

[31] A. S. Hassan, T. S. Hafez, Journal of Applied Pharmaceutical Science 8(5) (2018) 156-165.

[32] S. Li, Z. Wang, Y. Wei, C. Wu, S. Gao, H. Jiang, X. Zhao, H. Yan, X. Wang, Biomaterials 34(4) (2013) $902-$ 911.

[33] H. Benamara, T. Lanez, Journal of Fundamental and Applied Sciences 11(3) (2019) 1267-1278.

[34] A. Adaika, T. Lanez, E. Lanez, Journal of Fundamental and Applied Sciences 11(2) (2019) 748-768.

[35] E. Lanez, L. Bechki, T. Lanez, Chemistry and Chemical Technology 13(1) (2019) 11-17.

[36] X. Gao, G. Gong, Z. Zhang, G. Du, Y. Cao, G. Zhao, Journal of Molecular Structure 1200 (2020) 127077. https://doi.org/10.1016/j.molstruc.2019.127077

[37] S. Sansook, E. Lineham, S. Hassell-Hart, G. J. Tizzard, S. J. Coles, J. Spencer, S. J. Morley, Molecules 23(9) (2018) 2126. https://doi.org/10.3390/molecules23092126.

[38] S. Realista, S. Quintal, P. N. Martinho, A. I. Melato, A. Gil, T. Esteves, M. de Deus Carvalho, L. P. Ferreira, P. D. Vaz, M. J. Calhorda, Journal of Coordination Chemistry 70(2) (2017) 314-327.

[39] T. Lanez, P. L. Pauson, Journal of the Chemical Society, Perkin Transactions 1 (1990) 2437-2442.

[40] A. Ray, B. Koley Seth, U. Pal, S. Basu, Spectrochimica Acta - Part A: Molecular and Biomolecular Spectroscopy 92 (2012) 164-174.

[41] M. Frisch, G. Trucks, H. Schlegel, G. E. Scuseria..., Gaussian 09, Gaussian Inc., Wallingford, 2009.

[42] B. Miehlich, A. Savin, H. Stoll, H. Preuss, Chemical Physics Letters 157(3) (1989) 200-206.

[43] A. D. Becke, The Journal of Chemical Physics 98(7) (1993) 5648-5652. 
[44] G. M. Morris, H. Ruth, W. Lindstrom, M. F. Sanner, R. K. Belew, D. S. Goodsell, A. J. Olson, Journal of Computational Chemistry 30(16) (2009) 2785-2791.

[45] O. Trott, A. J. Olson, Journal of Computational Chemistry 31(2) (2009) 455-461.

[46] S. Bi, L. Yan, B. Wang, J. Bian, Y. Sun, Journal of Luminescence 131(5) (2011) 866-873.

[47] M. Mahanthappa, B. G. Gowda, J. I. Gowda, R. Rengaswamy, Journal of Electrochemical Science and Engineering 6(2) (2016) 155-164.

[48] J. B. M. Leuna, S. K. Sop, S. Makota, E. Njanja, T. C. Ebelle, A. G. Azebaze, E. Ngameni, A. Nassi, Bioelectrochemistry 119 (2018) 20-25.

[49] S. Pramanik, R. Chakraborty, International Journal of Biosensors \& Bioelectronics 2(3) (2017) 96-98.

[50] J. Wu, S. Y. Bi, X. Y. Sun, R. Zhao, J. H. Wang, H. F. Zhou, Journal of Biomolecular Structure and Dynamics 37(13) (2019) 3496-3505.

[51] J. R. Flores, R. O'Kennedy, M. R. Smyth, Analytica Chimica Acta 212 (1988) 355-358.

[52] A. E. M. Radi, S. H. Eissa, Eurasian Journal of Analytical Chemistry 6(1) (2011) 13-21.

[53] X. Chu, G. L. Shen, J. H. Jiang, T. F. Kang, B. Xiong, R. Q. Yu, Analytica Chimica Acta 373(1) (1998) 2938.

[54] M. Aslanoglu, G. Ayne, Analytical and Bioanalytical Chemistry 380 (2004) 658-663.

[55] C. M. A. Brett, A. N. A. Maria, O. Brett, Electrochemistry: Principles, Methods, and Applications, Oxford University Press, Oxford, UK, 1993, p. 444.

[56] M. Nie, Y. Wang, H. L. Li: Polish Journal of Chemistry 71(6) (1997) 816-822.

(C2020 by the authors; licensee IAPC, Zagreb, Croatia. This article is an open-access article distributed under the terms and conditions of the Creative Commons Attribution license (https://creativecommons.org/licenses/by/4.0/) 